\title{
Changements dans l'occupation du sol et l'aménagement des paysages du Mont-Liban du Néolithique aux époques historiques
}

Changes in land-use and management of Mount Lebanon's landscapes from the Neolithic to the historical periods

Romana Harfouche, Pierre Poupet, Chadi Abdallah, Linda Herveux, Corine Yazbeck, Ghaleb Faour, Johnny Samuele Baldi, Jwana Chahoud, Mohammad Abboud, Grace Homsy-Gottwalles, Tania Zaven et Pascal Verdin

\section{1 - Le bassin versant du Nahr Ibrahim et l'hinterland de Jbayl}

\section{1 - Historique du projet et espace d'étude}

Une recherche interdisciplinaire a été développée sur la vallée du Nahr Ibrahim, au sein du programme de coopération scientifique franco-libanaise CEDRE (2010-2012), entre le Centre national de la recherche scientifique français et le Centre de télédétection satellitaire du Conseil national de la recherche scientifique du Liban. Le projet CEDRE, intitulé "Agriculture et paysages dans la montagne libanaise à l'Holocène : protection et gestion durable des ressources naturelles en sol et en eau", avait pour objectif de combler une lacune scientifique concernant les paysages emblématiques du Liban. En effet, les territoires de la montagne libanaise n'ont jamais fait l'objet d'une étude de co-évolution des sociétés et du milieu physique sur le temps long, associant des données de l'archéologie, de l'histoire, de l'ethnologie, des sciences de la Terre et du sol, de la paléobotanique et de l'écologie végétale. Cette recherche, basée sur les travaux antécédents (Harfouche et Poupet, 2001), a permis de réaliser les premières analyses 
physico-chimiques sur les paléosols des terrasses agricoles anciennes ainsi que les premières analyses paléobotaniques dans la montagne libanaise (Harfouche et poupet, 2015). Depuis 2014, le projet "Nahr Ibrahim" est conduit dans le cadre d'une nouvelle programmation avec les partenaires institutionnels français (ministère de l'Europe et des Affaires étrangères; laboratoire ArScAn UMR 7041 Cnrs; Institut français du Proche-Orient) et libanais (Direction générale des antiquités pour le ministère de la Culture; Université libanaise; Lebanese International University; Centre de télédétection du Cnrs-L et Association pour la protection du Jabal Moussa).

2 Le Mont-Liban est un terrain d'étude privilégié, parce que ce vaste territoire très accidenté demeure peu connu, au regard des importantes investigations géoarchéologiques sur les cités-États, les tells et les ports du littoral, des études palynologiques relatives au Quaternaire dans la plaine de la Béqaa (Hajar, et al., 2010 ; Chedadi et Khater, 2016) des recherches paléosismiques et multi-proxy dans le polje de Yammouneh (Daeron, et al., 2007 ; Develle, et al., 2010 ; Gasse, et al., 2011 ; 2015) ainsi que des recherches paléoclimatiques à partir des spéléothèmes des grottes de Kanaan et de Jiîta (Verheyden, et al., 2008 ; Nehmé, et al., 2015 ; Cheng, et al., 2015). Le terrain d'étude est constitué par le bassin versant du Nahr Ibrahim, un des fleuves majeurs de la façade méditerranéenne du Mont-Liban, dont l'estuaire se situe à $7 \mathrm{~km}$ environ au sud de la ville portuaire plurimillénaire de Byblos/Jbayl (fig. 1A).

Figure 1

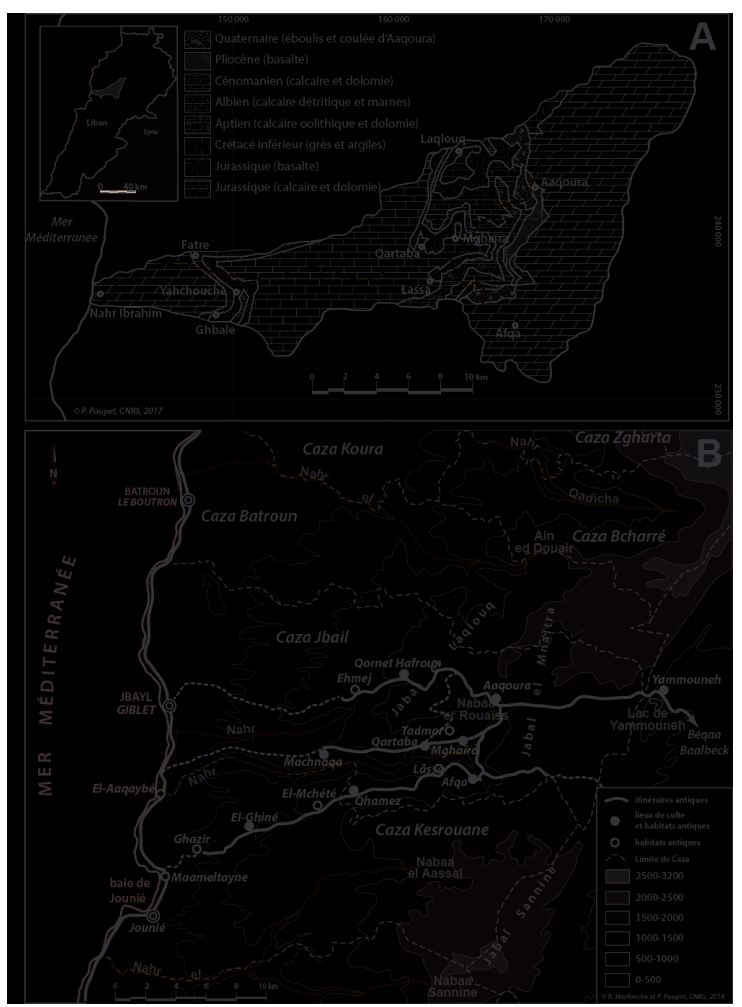

A- Carte géologique schématique du bassin versant du Nahr Ibrahim. B- Carte orohydrographique du Liban entre Nahr ej-Jaouz et Nahr el-Kelb avec les routes restituées à partir des sources écrites et archéologiques autour du Nahr Ibrahim, reliant le littoral à la Béqaa.

Crédits : (A) P. Poupet, Cnrs 2017 ; (B) R. Harfouche et P. Poupet, Cnrs 2014

3 Ce fleuve, l'Adonis de l'Antiquité, occupe une position médiane de l'arrière-pays de Byblos, entre deux fleuves : le Nahr ej-Jaouz au nord et le Nahr el-Kelb au sud. Ce réseau 
hydrographique prend une importance particulière dans les recherches sur l'exploitation des cèdres du Mont Liban et leur transport par voie d'eau (Elayi, 1988 ; Fontan, 2001). Mais le débit et la morphologie de ces cours sont autant d'obstacles à l'hypothèse de flottage des grumes : le Nahr ej-Jaouz a un débit de 65 millions de $\mathrm{m}^{3}$ par an, pour un bassin versant de $196 \mathrm{~km}^{2}$; le Nahr el-Kelb a un débit plus important, de $250 \mathrm{Mm}^{3} /$ an pour un bassin versant de $231 \mathrm{~km}^{2}$; occupant l'espace entre les deux précédents, le Nahr Ibrahim a un débit de $395 \mathrm{Mm}^{3}$ /an pour un bassin versant de $326 \mathrm{~km}^{2}$ (Shaban et Hamze, 2017). Par ailleurs, le bassin versant du Nahr Ibrahim est un axe majeur de pénétration dans la montagne, qui relie Byblos à la Béqaa et à Héliopolis/ Baalbeck, en traversant les plateaux calcaires sommitaux vers $2000 \mathrm{~m}$ d'altitude (fig. 1B). Le court fleuve pérenne coule d'est en ouest, depuis la source d'Afqa vers $1210 \mathrm{~m}$ d'altitude. Les reliefs, principalement calcaires et calcaro-marneux, sont pentus et présentent de nombreux dénivelés abrupts. Grâce à sa position géographique, le bassin versant bénéficie de conditions climatiques méditerranéennes et dispose de ressources en eau remarquables dans le contexte proche-oriental. Les précipitations moyennes s'élèvent à $1030 \mathrm{~mm} / \mathrm{an}$ (Shaban, et al., 2015) et la neige couvre la haute montagne durant la saison hivernale (décembre à mars). La couverture végétale du bassin versant du Nahr Ibrahim est constituée essentiellement de la forêt méditerranéenne. Cette couverture, plus ou moins anthropisée, est majoritairement composée d'une mosaïque de forêts et de garrigues $(30 \%)$ ainsi que de pelouses en haute altitude (25\%) (Abdallah, et al., 2015). Les essences dominantes des formations boisées, denses ou clairsemées, sont celles de la chênaie mixte sempervirens et caducifoliée (Quercus calliprinos et Q. infectoria), associée à des conifères (Pinus sp., Juniperus sp., Cupressus sp.) et au pistachier (Pistacia lentiscus).

4 Tous ces paramètres ont permis le développement de sols riches, malgré le caractère accidenté du relief (Harfouche et Poupet, 2009). Dans les zones où les sols sont les plus favorables à l'agriculture, les paysages offrent des aménagements de terrasses agricoles pluriséculaires. Cette étude démontre que les paysages ont été progressivement bâtis par des communautés agropastorales, selon au moins quatre phases majeures de (ré)aménagement des versants : i) au début du III millénaire, ii) à l'époque romaine/ protobyzantine, iii) aux XII-XIII ${ }^{\mathrm{e}}$ siècles iv) et au cours d'un Moyen Âge tardif (Harfouche et Poupet, 2002 ; Harfouche, 2007).

5 Un autre paramètre majeur de la morphologie paysagère de la zone d'étude est celui de la tectonique (fig. 2). 
Figure 2



Carte des accidents tectoniques au Liban

P. Poupet (2015) d'après C. D. Walley, LSB 10-1, 1997, fig.2, p.18)

6 En effet, la structure commande les voies de communication, préférentiellement sur les interfluves, et la localisation de l'habitat qui se développe principalement sur les replats et les pentes modérées au-dessus des à-pics rocheux. La région est depuis longtemps marquée par une tectonique active, notamment par l'activité de la faille de Yammouneh, qui est la prolongation nord du rift africain. Le rayon d'action des séismes qu'elle engendre est évalué à une dizaine de kilomètres, couvrant ainsi la haute vallée du Nahr Ibrahim (Daeron, et al., 2007). Le séisme le plus ancien est attesté par une correspondance entre le roi de Tyr, Abimilki, et Aménophis IV (Akhenaton), mentionnant la destruction d'Ougarit (incendie et tsunami) et de Byblos en $1365 \mathrm{BC}$ (Guidoboni, et al., 1994). D'autres séismes, souvent accompagnés de tsunamis et de glissements de terrain, ont affecté le Mont Liban et le littoral libanais (Sbeinati, et al., 2005). Parmi eux, celui de 349 AD et celui de 1202 AD ont bien été enregistrés dans les dépôts du paléolac de Yammouneh (Daeron, et al., 2007). Les mouvements de terrain et l'érosion des sols, telle la célèbre coulée de boue d'Aaqoura avec ses deux épisodes récents en 1929 et 1937, figurent parmi les risques majeurs auxquels ont été confrontées les populations installées sur les versants montagneux du Nahr Ibrahim (Abdallah, et al., 2015). Ce facteur d'instabilité des pentes, très actif dans la haute vallée, a non seulement des conséquences sur la répartition de l'habitat et des champs, mais il a aussi participé à la construction des représentations religieuses et socio-économiques de la montagne dans l'Antiquité. Ainsi, la purge des limons argileux rouges piégés dans le karst, très développé dans les formations calcaires, lors de la fonte du manteau neigeux, renvoie à un phénomène géopédologique et hydrologique responsable de la teinte rouge des eaux du fleuve (Harfouche, et al., 2015a). 
7 Tous ces faits liés à la géologie et à la géomorphologie sont essentiels à l'archéologie des paysages et l'installation des sociétés humaines, car pour restituer l'évolution des formes du peuplement, il faut comprendre les conditions d'habitabilité de la montagne et les mécanismes de l'érosion sur les versants. Ces mécanismes sont étroitement imbriqués aux faits anthropiques dans les stratifications archéologiques. La démarche de cette étude confère une place à part entière aux processus et aux éléments naturels d'une histoire territorialisée, où les cartes aident à mieux comprendre les milieux comme acteurs.

\section{2 - Problématique et méthode}

8 Le projet d'étude "Nahr Ibrahim" s'attache à caractériser les formes de peuplement depuis la fin du Pléistocène et les impacts sur l'environnement forestier, les sols et les ressources en eau de la construction progressive des paysages en terrasses par les premières communautés d'agriculteurs depuis la culture pré-néolithique du Natoufien $(\sim 12000 \mathrm{BC})$ jusqu'à l'actuel, en passant par les époques des complexifications sociales et des mutations socio-économiques de l'âge du Bronze (Huot, 2004). Les premières recherches ont confirmé le potentiel de cette région sans masquer les carences, en particulier la méconnaissance des sites archéologiques qui obère la reconstitution diachronique de la répartition du peuplement pré-moderne dans la montagne libanaise. La recherche s'articule autour de trois axes thématiques. Le premier concerne le peuplement et les formes de l'habitat: quelles sont les traces les plus anciennes et sous quelles formes ces installations ont évolué au cours du temps? S'agitil d'habitat permanent ou saisonnier? Avec une organisation groupée ou dispersée ? Le deuxième axe de recherche concerne la restitution des paléoenvironnements et la construction des territoires par la mise en place des réseaux d'habitats et de circulation, ainsi que l'organisation des parcellaires en terrasses qui marque la mise en valeur agricole durable de la montagne. Nos travaux ayant déjà souligné la continuité du peuplement, sans hiatus majeur (Harfouche, et al., 2015a, 2017), peut-on malgré cela identifier des périodes de plus faible intensité dans l'occupation de la montagne? Comment se traduisent-elles au plan de l'aménagement du territoire? Quelles sont les modalités de ces changements, au regard de l'imbrication des variables naturelles et socio-économiques dans ce milieu contraignant? Le troisième axe porte sur l'exploitation des ressources (minérales, végétales, animales), les productions (agricoles et artisanales) et enfin, l'économie de montagne avec ses liaisons vers le littoral et le nord-est du bassin méditerranéen, le Levant Sud et au-delà jusqu'à l'Égypte, ainsi que vers la Béqaa, la Syrie septentrionale et la Mésopotamie.

9 L'étude proposée consiste en une évaluation archéologique et diachronique du territoire à partir de prospections régionales pédoarchéologiques, d'un récolement bibliographique et de fouilles sous la forme de sondages ciblés sur des sites d'habitat ayant une épaisseur pédosédimentaire importante. La définition des transitions et les temporalités des changements sont examinées à partir des données archéologiques et historiques, radiochronologiques $\left({ }^{14} \mathrm{C}\right)$, géologiques et pédologiques (paléosols), paléobotaniques (anthracologie, carpologie, phytolithes), ethnologiques et iconographiques (Harfouche, 2003). 


\section{2 - L'occupation du sol entre le Nahr el-Kelb et le Nahr ej-Jaouz}

\section{1 - La base de données}

La cartographie du peuplement entre le Nahr el-Kelb et le Nahr ej-Jaouz a été réalisée à partir d'un dépouillement bibliographique le plus exhaustif possible pour localiser les sites connus, datés, et nouvellement prospectés (Harfouche, et al., 2017). II convient de souligner d'emblée plusieurs limites de notre étude. La première concerne la faible résolution des données chronologiques des sites. En effet, le tableau de l'occupation de l'arrière-pays de Byblos, constitué de grandes étapes chronologiques successives, est marqué par des limites temporelles floues. Presque aucun site n'est correctement daté, en l'absence de sondages élargis et systématiques; dans la plupart des cas, seuls quelques mobiliers collectés en surface fournissent des repères chronologiques approximatifs. Dans la mesure où la grande majorité de ces sites n'a fait l'objet d'aucune fouille archéologique exhaustive et bien datée, leur cartographie a été effectuée selon les larges périodes chrono-culturelles conventionnelles (Tab. 1).

Tableau 1

\begin{tabular}{|c|c|c|}
\hline $\begin{array}{l}\text { DATE BC } / \\
\text { AD }\end{array}$ & CHRONOCULTURES & SITES LIBANAIS / REPÈRES HISTORIQUES \\
\hline 1517-1918 & époque ottomane & $\begin{array}{l}\text { 1517: Les Ottomans chassent les Mameloukes } \\
\text { égyptiens }\end{array}$ \\
\hline \multirow{4}{*}{$636-1517$} & \multirow{4}{*}{ Moyen Âge } & 1291 : Sultanat mamelouke \\
\hline & & $\begin{array}{l}1102 \text { : États latins d'Orient (Comté de Tripoli dans la } \\
\text { moitié nord du Liban) }\end{array}$ \\
\hline & & Califats omeyyade, puis abbasside/fatimide \\
\hline & & 636 : conquête arabo-musulmane \\
\hline 395-636 & époque byzantine & En 395 le Liban passe sous domination byzantine \\
\hline $\begin{array}{ll}333 & B C-395 \\
A D & \end{array}$ & époque gréco-romaine & $\begin{array}{l}\text { Chute de Tyr en } 332 \text { BC (Alexandre le Grand), puis } \\
\text { création de la Province romaine de Syrie en } 64 \text { AD }\end{array}$ \\
\hline $1200-333 \mathrm{BC}$ & âge du Fer & $\begin{array}{l}\text { Cités phéniciens indépendantes, puis sous } \\
\text { dominations assyrienne, égyptienne, perse, } \\
\text { babylonienne, grecque }\end{array}$ \\
\hline $1550-1200$ & âge du Bronze Récent & Développement des cités- États sur la côte (Byblos, \\
\hline $2000-1550$ & âge du Bronze Moyen & Loz) \\
\hline $3500-2000$ & âge du Bronze Ancien & Byblos, Saïda, Tell Arqa, Tell Fadous, Tell el Ghassil \\
\hline
\end{tabular}




\begin{tabular}{|l|l|l|}
\hline $5300-3500$ & $\begin{array}{l}\text { Chalcolithique Récent-Final } \\
\text { Chalcolithique Moyen }\end{array}$ & $\begin{array}{l}\text { Byblos, Minet Dalieh, Jisr, Dakerman, Tell Ard Tlaili, } \\
\text { Kroum et Thatné }\end{array}$ \\
\hline $5800-5300$ & $\begin{array}{l}\text { Chalcolithique Ancien } \\
\text { Néolithique Récent }\end{array}$ & Moukhtara, Byblos, Jiita, Dakerman, Tell Ard Tlaili \\
\hline $6900-5800$ & Néolithique Ancien & Arslan, Byblos, Nabaa Faour, Labwé \\
\hline $8000-6900$ & Pre-Pottery Neolithic B (PPNB) & $\begin{array}{l}\text { Byblos, Tell Hmaira, Sables de Beyrouth-Tell aux } \\
\text { Scies, Dik el Mehdi, Labwé, Saaidé I, Nachcharini }\end{array}$ \\
\hline $9500-8800$ & Pre-Pottery Neolithic A (PPNA) & Sables de Beyrouth, Borj Barajné, Nachcharini \\
\hline $12000-9500$ & Natoufien & $\begin{array}{l}\text { Jiita III, Jiyé, Borj Barajné, Ain Chaub, Jebel Saaidé, } \\
\text { Nachcharini, Ammiq II }\end{array}$ \\
\hline
\end{tabular}

Grandes divisions chrono-culturelles dans la région étudiée

11 La deuxième limite concerne la signification de la représentation spatiale. Cette forme de représentation spatiale et diachronique met en avant un niveau de lecture privilégiant la perception d'une continuité dans l'occupation d'un site en raison de la faible résolution des données chronologiques. Seule la fouille archéologique serait à même de révéler des ruptures spatio-temporelles dans l'occupation de ces habitats. La troisième limite est étroitement liée à la nature intrinsèque de la documentation. Les cartes produites reflètent d'abord un état des connaissances représentatif des centres d'intérêt des chercheurs et de leurs choix géographiques. C'est en ce sens qu'il faut comprendre le déséquilibre patent qui apparaît, avec des concentrations de points dans quelques secteurs et des vides dans d'autres. Si d'évidentes distorsions sont intimement liées à la nature de la documentation, quelques traits majeurs se dégagent néanmoins dans l'évolution spatiale de l'occupation et de l'histoire du peuplement.

\section{2 - Les résultats, du Néolithique au Moyen Âge}

12 Nos recherches s'attachent plus particulièrement à déterminer la première mise en place des sociétés d'agriculteurs en montagne. Les nombreuses références bibliographiques - provenant d'une documentation dispersée - sont relatives à une centaine de sites que compte la base de données (Harfouche, et al., 2017). Aux époques Néolithique-Chalcolithique $(\sim 12000 \mathrm{BC}$ à $3500 \mathrm{BC})$, les sites connus sont plutôt distribués sur le littoral (fig. 3). 
Figure 3

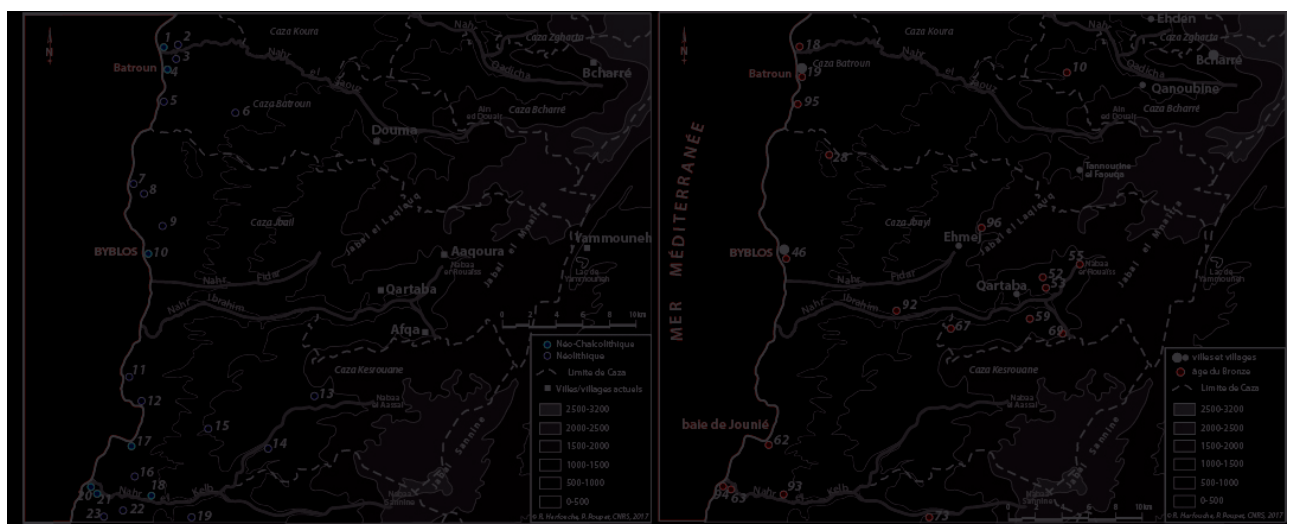

À gauche, carte des principaux sites connus du Néolithique et du Chalcolithique entre le Nahr el-Kelb au sud et le Nahr ej-Jaouz au nord. À droite, carte des sites de l'âge du Bronze

Crédits : R. Harfouche et P. Poupet, Cnrs 2017

Quelques rares sites plus en altitude sont répertoriés de part et d'autre du Nahr el-Kelb. L'âge du Bronze marque l'émergence des grandes cités côtières. La distribution des 19 sites correspond aux aires les plus intensément prospectées : la région de Batroun et le débouché du Nahr ej-Jaouz, la haute vallée du Nahr Ibrahim et la basse vallée du Nahr el-Kelb. Treize sites seulement sont répartis dans le même espace à l'âge du Fer (fig. 4A).

\section{Figure 4}

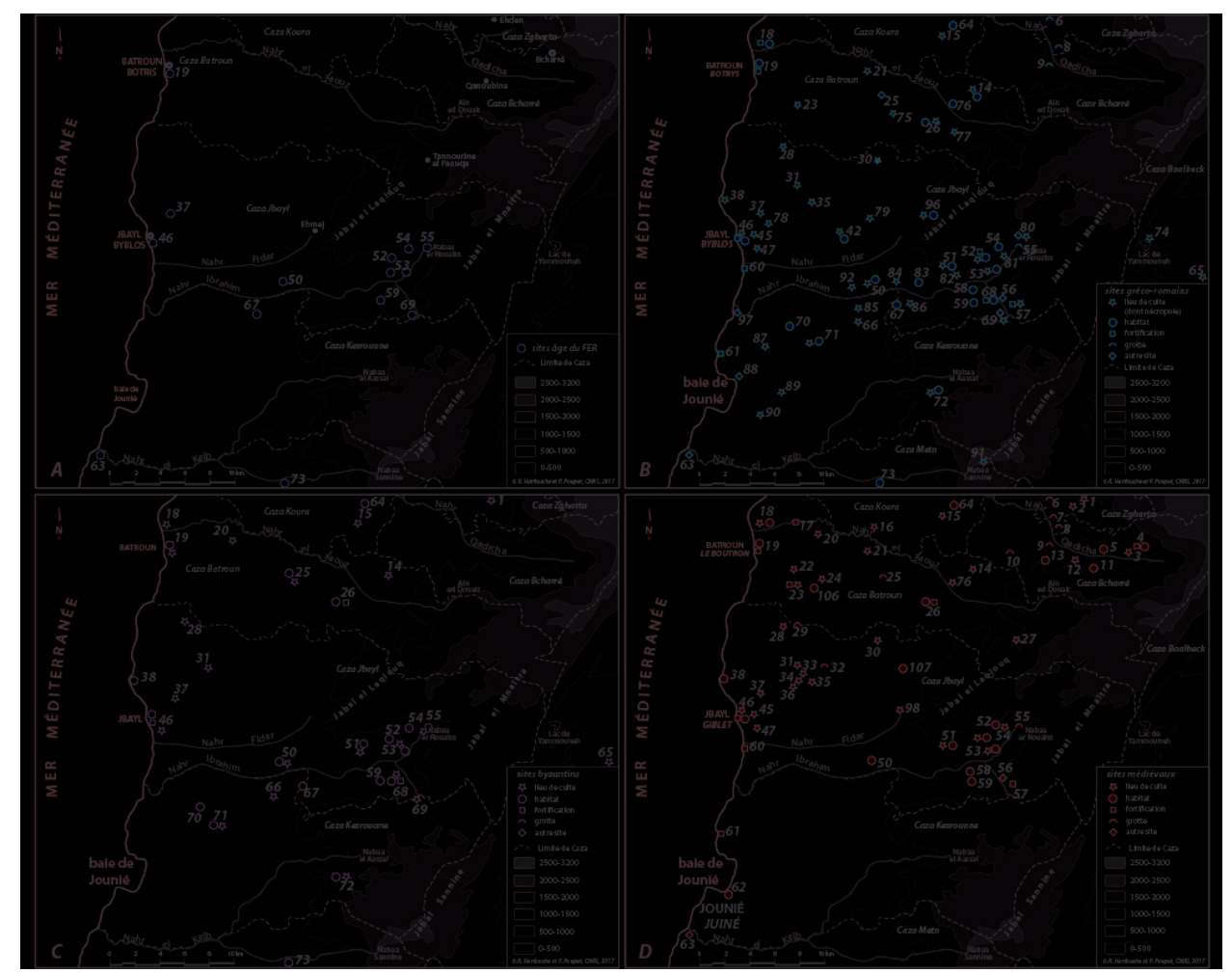

(A) Carte des sites de l'âge du Fer. (B) Carte des sites gréco-romains. (C) Carte des sites byzantins. (D) Carte des sites médiévaux, ne comprenant pas les casaux.

Crédits : R. Harfouche et P. Poupet, Cnrs 2017 
Hormis quelques sites côtiers qui existaient déjà à l'âge du Bronze, la concentration des sites dans la haute vallée du Nahr Ibrahim s'explique par une activité de prospection préférentielle dans cette région, au début des années 2000. L'augmentation spectaculaire à 61 sites pour l'époque gréco-romaine est due à une surreprésentation des lieux de culte; des temples auxquels sont associées les nécropoles (fig. 4B). De plus, le mobilier céramique (tuiles, amphores...) est plus résistant à l'érosion, les sites sont moins enfouis et surtout facilement identifiables par les archéologues, à la fois au regard des vestiges architecturaux et de la connaissance du mobilier céramique. À l'époque byzantine, le nombre de sites connus se réduit de moitié jusqu'à 30 sites (fig. 4C). Là encore, la cartographie représente davantage l'état des connaissances des sites dans les zones prospectées que la réalité des espaces habités. D'ailleurs, une continuité d'occupation en ressort sur la plupart des sites depuis l'époque romaine jusqu'au début du christianisme. Une tendance distinctive semble néanmoins se dessiner : l'abandon des sites de plein air qui étaient autrefois fréquentés aux altitudes les plus élevées, au-dessus de $1700 \mathrm{~m}$. Pour l'époque médiévale, le centre de gravité des recherches bascule clairement vers le nord, comme le montre l'absence de sites dans le caza du Kesrouane (fig. 4D). Tout comme la carte de l'époque gréco-romaine, la carte médiévale est particulièrement représentative de la croissance des activités de recherche, en particulier celles sur les églises rurales, dont les églises peintes au nord du Nahr Ibrahim. Il faut ajouter les textes croisés qui mentionnent des casaux (dépendances rurales soumises à redevances), dont certains ont pu être localisés dans la toponymie actuelle.

Face à une représentation spatiale disparate, montrant malgré tout une tendance à tout le moins d'un abandon de sites en plein air à haute altitude vers les sites à plus basse altitude, un changement d'échelle et de protocole d'intervention s'impose donc pour pallier les carences de la documentation et progresser dans la connaissance diachronique des paysages et des territoires.

\section{3 - L'évolution du peuplement et des paysages dans la haute vallée du Nahr Ibrahim}

\section{1 - L'implantation de l'habitat dans le bassin versant}

16 Hormis les multiples sondages archéologiques sur le sanctuaire gréco-romain et la basilique paléochrétienne de Mghaïra-Yanouh, pour reconnaître et dater les différentes phases architecturales, les sites d'habitat archéologiques ne sont connus que par des prospections ciblées à partir d'informateurs locaux, dans la haute vallée du Nahr Ibrahim et sur le versant méridional de la moyenne vallée dans le Jabal Moussa (Gatier et Nordiguian, 2005; Doumet-Serhal, 2010). Vingt-cinq sites archéologiques ont été répertoriés dans le bassin versant du Nahr Ibrahim, auxquels il faut ajouter un site identifié sur des bases linguistiques à une localité existante (Es-Snawbar) à partir d'une mention dans une charte latine médiévale de 1143, mais sans aucun vestige au sol reconnu et daté (Harfouche, et al., 2017) (fig. 5). 




Carte de localisation des sites retenus dans le bassin versant du Nahr Ibrahim : 50. El Machnaqa ; 51 Hsaïya ; 52. Tadmor ; 53. Mghaïra-Yanouh ; 54. Mar Edna ; 55. Mar Hanna ; 56. El Burj-El Mnaïtra ; 57. El Hosn/Le Moinestre? ; 58. Qalaa ; 59. Lâssa ; 66. Hidchet ; 67. Broqta ; 68. El Mzârib ; 69. Afqa ; 80. Aaqoura ; 81. Mar Mansour ; 82. Qartaba ; 83. Qalaat Nemroud ; 84. Frat ; 85. Yahchouch ; 86. Qahmez ; 92. Meshane ; 97. Nahr Ibrahim (pont) ; 101. Es Snawbar (casal) ; A. Nahr Ibrahim (grotte) ; B. Adonis Achtrout. Système de coordonnées : projection conique conforme de Lambert, système Levant.

Crédits : R. Harfouche, 2016

17 Par ailleurs, ce nombre ne comprend pas les multiples aménagements rupestres liés à la transformation des fruits (fouloirs-pressoirs, citernes) qui sont encore visibles sur l'ensemble du bassin versant. Leur datation est difficile à établir en l'absence de recouvrement sédimentaire, d'autant plus qu'ils ont souvent une utilisation pluriséculaire et que les fouloirs rupestres sont la plus ancienne forme d'installation pour l'extraction des jus de raisin et de l'huile d'olive connue au Proche-Orient, au moins depuis l'âge du Bronze (Brun, 2004).

18 Si l'on considère la nature des sites, trois grottes sont occupées $(A, B, 55)$, deux étant situées de part et d'autre de l'embouchure du fleuve et une au niveau des sources du Nahr Rouaïss (Tab. 2).

Tableau 2

\begin{tabular}{|l|l|l|l|l|l|l|l|}
\hline $\mathbf{N}^{\circ}$ & Toponymes & Catégorie & Br & Fe & G-R & Byz & MA \\
\hline 50 & El Machnaqa & CH & & & & & \\
\hline 51 & Hsaïya & HC & & & & & \\
\hline 52 & Tadmor & HCF & & & & & \\
\hline
\end{tabular}




\begin{tabular}{|c|c|c|c|c|c|}
\hline 53 & Mghaïra-Yanouh & $\mathrm{HC}$ & & & \\
\hline 54 & Mar Edna & $\mathrm{H}$ & & & \\
\hline 55 & Mar Hanna & G & & & \\
\hline 56 & El Burj-El Mnaïtra & AS & & & \\
\hline 57 & El Hosn & HFC & & & \\
\hline 58 & Qalaa & $\mathrm{H}$ & & & \\
\hline 59 & Lâssa & $\mathrm{H}$ & & & \\
\hline 66 & Hidchet & $\mathrm{HC}$ & & & \\
\hline 67 & Broqta & $\mathrm{H}$ & & & \\
\hline 68 & El Mzârib & $\mathrm{HF}$ & & & \\
\hline 69 & Afqa & CAS & & & \\
\hline 80 & Aaqoura & ASC & & & \\
\hline 81 & Mar Mansour & c & & & \\
\hline 82 & Qartaba & C & & & \\
\hline 83 & Qalaat Nemroud & $\mathrm{H}$ & & & \\
\hline 84 & Frat & C & & & \\
\hline 85 & Yahchouch & C & & & \\
\hline 86 & Qahmez & C & & & \\
\hline 92 & Meshane & C & & & \\
\hline 97 & Nahr Ibrahim (pont) & AS & & & \\
\hline 101 & Es Snawbar (casal) & $\mathrm{H}$ & & & \\
\hline A & Nahr Ibrahim & G & Paléolit & hique moyen & \\
\hline B & Adonis Achtrout & G & Paléolit & hique moyen & \\
\hline \multicolumn{6}{|c|}{$G=$ Grotte $; \mathrm{H}=$ Habitat $; \mathrm{C}=$ Cultuel $; \mathrm{F}=$ Fortification $; \mathrm{AS}=$ Autre site } \\
\hline
\end{tabular}

Sites retenus dans le bassin versant du Nahr Ibrahim selon les grandes divisions chrono-culturelles

Des espaces funéraires ont été reconnus dans onze localités $(50$ à $53 ; 57 ; 66 ; 80 ; 81$; $84 ; 86 ; 92)$ et treize sites ont livré des traces d'habitat plus ou moins explicites (50 à 
$54 ; 57$ à $59 ; 66$ à $68 ; 83 ; 86$ ), auxquels il faut ajouter un casal connu uniquement par une charte du XII siècle (101). On peut également supposer que le site d'Aaqoura (80), où un espace funéraire antique a été identifié, comptait un habitat à proximité. La question du site d'Afqa est impossible à démêler en raison de la méconnaissance de ce lieu, célèbre dans les sources écrites de l'Antiquité, mais qui n'a jamais fait l'objet de fouilles archéologiques extensives. La seule certitude est que les habitats implantés sur les hauts des versants de la vallée du Nahr Ibrahim représentent $65 \%$ de la totalité des sites de plein air recensés qu'ils soient sédentaires, précaires, temporaires ou mobiles. Ce pourcentage élevé rompt définitivement l'image de la montagne comme marge impénétrable et hostile, éventuellement occupée de manière sporadique et ne pouvant être habitée durablement.

Au plan chronologique, hormis les deux grottes sur le littoral (grotte d'Adonis Achtrout et grotte du Nahr Ibrahim/Aasfouriyé) occupées au Paléolithique moyen, la montagne n'a pas livré de traces d'installations antérieures au III ${ }^{\mathrm{e}}$ millénaire BC, si ce n'est des charbons de bois dans un profil pédologique polygénique identifié et donnant une indication sur la présence humaine à Mghaïra au début du $\mathrm{V}^{\mathrm{e}}$ millénaire (Harfouche, et al., 2015a). Par contre, aucune datation radiochronologique n'a été obtenue pour le II ${ }^{\mathrm{e}}$ millénaire.

Dans la haute montagne, au pied du Jurd (terme arabe qui désigne les terres au couvert végétal très dégradé des plateaux sommitaux), en amont des sources du Nahr Rouaïss et du Nahr Afqa, quelques découvertes anciennes prouvent que la montagne était déjà parcourue dès la Préhistoire. En 1947, A. Bergy découvrait le site d'Aaqoura (80) daté du Paléolithique moyen, sur un sentier conduisant à Afqa, lieu bien difficile à localiser aujourd'hui (Copeland et Wescombe, 1965). Espace à forte contrainte climatique, la montagne n'aurait été, selon les préhistoriens, qu'un lieu de fréquentation épisodique, ponctué de campements saisonniers sur la route des exploitations de matières premières siliceuses, pour les habitants de la Béqaa. Aucun site néolithique et chalcolithique n'a été répertorié dans le bassin versant du Nahr Ibrahim. La tombe localisée dans la moyenne vallée, à Meshane (92), qui serait datée du IV millénaire, n'a pas été publiée et demeure un unicum au plan chronologique. L'occupation la plus ancienne est datée de l'âge du Bronze Ancien II et correspond à l'habitat de Mghaïra-Yanouh (53). C'est également à l'âge du Bronze Ancien qu'apparaissent trois autres habitats $(52 ; 59 ; 67)$ et que la grotte Mar Hanna (55) est occupée. Ces mêmes lieux sont encore habités à l'âge du Fer. Il faut ajouter un site (54) dans la haute vallée et probablement celui d'Afqa (69), si l'on considère l'ancienneté du culte qui y était pratiqué. Mais l'âge du Fer est une période encore méconnue en montagne. Tous les sites archéologiques répertoriés dans le bassin versant sont occupés à l'époque grécoromaine, en sachant que les espaces funéraires constitués par les nombreuses nécropoles rupestres ont été inclus. Elles sont difficiles à dater et reçoivent des défunts à différentes époques antérieurement au Moyen Âge, au moins depuis le III millénaire. L'époque protobyzantine est plutôt une période de continuité que de rupture pour l'habitat. Enfin, sur les vingt-trois sites retenus dans l'inventaire depuis la Préhistoire récente, seuls neuf sont signalés comme ayant livré du mobilier céramique et/ou des vestiges attribuables à l'époque médiévale (50 à $54 ; 56$ à 59), auxquels il faut ajouter une grotte (55) et un casal cité dans une charte datée de 1143 (101). Ce nombre relativement faible de sites est probablement dû à la manière dont ont été conduites les fouilles/prospections sur le terrain car aucun mobilier médiéval n'est signalé sur les 
sites prospectés dans le Jabal Moussa. Cette faible concentration s'explique par un biais lié à la conduite de la recherche archéologique qui a porté son attention plutôt sur les occupations antérieures. Il est certain que le nombre de hameaux/villages/casaux était plus important, mais l'absence de recherche archéologique spécifique à la période médiévale ne permet pas d'aller plus loin dans l'interprétation de la représentation spatiale.

Si la majorité des habitats présente une occupation pluriséculaire, seuls trois sites de plein air offrent des traces d'occupations plurimillénaires, de l'âge du Bronze à l'époque ottomane, tous implantés dans le haut bassin versant du Nahr Ibrahim: Tadmor (52), Mghaïra-Yanouh (53) et Lâssa (59). La grotte Mar Hanna (55) est fréquentée depuis l'âge du Bronze jusqu'au Moyen Âge. Dans la moyenne vallée, l'habitat de Broqta (67) aurait été occupé depuis l'âge du Bronze jusqu'à l'époque byzantine. Les autres habitats ne semblent pas avoir existé avant le tournant du I ${ }^{\text {er }}$ millénaire $\mathrm{BC}$ d'après le mobilier céramique identifié en prospection.

Comme nous l'avons souligné, les paysages du bassin versant du Nahr Ibrahim sont liés au substrat géologique où dominent les calcaires de lithologie variée, qui représentent environ $80 \%$ des affleurements rocheux. Cette variété pétrographique donne naissance à des paysages différents. Les calcaires jurassiques forment l'essentiel de la moyenne vallée, générant ainsi des paysages ruiniformes avec des sols très peu épais. Les principaux habitats sont implantés en limite $(50 ; 66)$ et à l'extérieur des calcaires jurassiques. La moyenne vallée est pourtant riche en nécropoles rupestres notamment à El Machnaqa (50) et à Frat (84) et n'est pas dépeuplée pour autant $(67 ; 83 ; 85 ; 86)$.

24 À l'ouest, la vallée se rétrécit et forme des escarpements importants en direction des calcaires du Crétacé. À l'est de la moyenne vallée s'étend la partie la plus riche du bassin versant. Elle est marquée par des replats importants où sont installés les habitats et des sols profonds aménagés en terrasses agricoles. Le site de Qartaba (82) est implanté sur les sols riches, à l'interface de ces deux unités paysagères différentes. La géologie des calcaires dans cette zone intermédiaire est variée, avec quelques épanchements volcaniques marqués par des sols profonds et fertiles. Cette diversité génère une mosaïque paysagère allant jusqu'aux hauts sommets à Laqlouq. Aucun site archéologique n'est en revanche connu plus en amont, sur les hauts plateaux asylvatiques dans le domaine du jurd.

\section{2 - L'approche croisée intra- et extra-site dans la haute vallée}

C'est donc le haut bassin versant qui a concentré nos recherches jusqu'à présent, car c'est là que les paysages de terrasses sont les moins dégradés et que les sols sont les plus profonds. Ils offrent un cadre optimal à la fouille archéologique de champ et d'habitat qui présentent les stratifications les plus épaisses et donc les plus riches en informations. Nous avons privilégié une "zone atelier" d'environ $70 \mathrm{~km}^{2}$ dans la haute vallée, depuis les sources des Nahr Rouaïss et Nahr Afqa (avec leur confluence formant le Nahr Ibrahim) jusqu'à l'agglomération de Qartaba.

Au plan méthodologique, le constat est le suivant : d'une part, la production de cartes de points-sites à partir d'informateurs locaux - qui sont bien souvent eux-mêmes les pilleurs des sites archéologiques - reste insuffisante d'autre part, la datation de l'occupation des sites repérés en s'appuyant uniquement sur la prospection en surface de fragments de céramiques n'est jamais le reflet exact au plan chronologique d'une 
stratification. Il s'agissait donc de conduire une fouille archéologique sur les structures agraires conjointement à la fouille de l'habitat. C'est en cela que réside l'originalité de cette recherche : l'étude interdisciplinaire de l'évolution du territoire habité en lien avec l'agrosystème. Celle-ci porte donc sur plusieurs objets qui s'articulent entre eux, à différentes échelles, du paysage à la parcelle, en passant par l'habitat.

L'étude menée hors site archéologique stricto sensu favorise la vision verticale de sections étudiées en coupe, singulièrement dans des sondages pédoarchéologiques derrière les murs de terrasses et implantés au sein des unités paysagères majeures de la haute vallée. La lecture pédologique macroscopique des sols et des paléosols en relation avec ces terrasses, réalisée sur le terrain, était suivie de prélèvements pour des analyses en laboratoire (sédimentologie, archéobotanique, datations ${ }^{14} \mathrm{C}$ par AMS). Il était donc nécessaire de compléter ces interventions en passant à la fouille archéologique pluridisciplinaire des sites les moins endommagés. Cette approche, complémentaire de l'archéologie agraire, est seule susceptible de permettre la caractérisation des formes de l'habitat et la datation des structures, ainsi que les prélèvements carpologiques et anthracologiques dans des unités stratigraphiques identifiées dans leur extension spatiale. Notre choix s'est porté sur l'habitat du Tell el-Kharayeb (Yanouh-Mghaïra), à $1200 \mathrm{~m}$ d'altitude, en raison de l'intérêt scientifique unique du lieu. Il s'agit d'un site archéologique majeur du bassin versant du Nahr Ibrahim, bien préservé et présentant des épaisseurs stratifiées de plusieurs mètres.

Les analyses paléobotaniques réalisées sur l'habitat peuvent ainsi être croisées avec les résultats de l'archéologie du champ qui était conduite sur les parcellaires en terrasses. Cette analyse croisée permet ainsi de progresser dans la connaissance des lieux de production agricole, de l'articulation des espaces cultivés aux zones non aménagées et exploitées pour la cueillette ainsi que pour l'approvisionnement en bois et en fourrage. Les phénomènes observés dans les stratifications ont été calés chronologiquement par les dates radiochronologiques. Celles-ci ont été réalisées sur des échantillons prélevés dans des contextes différents : i) séquence pédosédimentaire "hors-site archéologique", ii) paléosols de terrasses agricoles faisant l'objet de sondages pédoarchéologiques, iii) stratification archéologique intra-site (un habitat et sa nécropole) dans laquelle les phénomènes anthropiques et pédosédimentaires sont imbriqués. Quinze dates ont été obtenues sur la séquence du site du Tell el-Kharayeb et un total de vingt-sept dates ont été acquises depuis le début du projet CEDRE sur des séquences pédoarchéologiques réparties dans la haute vallée du Nahr Ibrahim (Harfouche, et al., 2015a , 2017).

Cette mise en perspective des données collectées hors-site et intra-site est fondamentale et nouvelle pour l'archéologie au Liban comparée à d'autres régions où elle a été appliquée (Jacomet, et al., 2016). Elle permet aussi de nuancer les effets induits par le type de données produites selon les lieux et les disciplines impliquées. Les vestiges collectés sur site sont communément étudiés dans une perspective de comportements économiques des sociétés, alors que la plupart des enregistrements hors-site permettent de tirer des conclusions sur l'évolution paléoenvironnementale.

\section{3 - Les premières étapes du peuplement et de la construction des paysages}

La première modification du couvert forestier dans le haut bassin versant du Nahr Ibrahim mise en évidence date du début du $V^{\mathrm{e}}$ millénaire (fig. 6). 
Figure 6



Interprétation pédologique du profil complexe de LBN-7 à Taht el-Horch, commune de Mghaïra (coordonnées géographiques : $34^{\circ} 10^{\prime} 49^{\prime \prime} \mathrm{N}-35^{\circ} 88^{\prime} 14^{\prime \prime E}-1178 \mathrm{~m}$ alt.). Le sol polygénique, constitué par la superposition de quatre phases de pédogenèse sur une épaisseur sédimentaire de plus de $4 \mathrm{~m}$, témoigne des relations sociétés-milieux au cours des sept derniers millénaires (les teintes utilisées pour visualiser le profil polygénique sont celles de la charte Munsell ; les codes des horizons sont ceux préconisés par le Référentiel pédologique français).

Un sol polygénique (LBN-7, commune de Mghaïra, au lieu-dit de Taht el-Horch; coordonnées géographiques: $34^{\circ} 10^{\prime} .49^{\prime \prime} \mathrm{N}-35^{\circ} 88^{\prime} 14^{\prime \prime} \mathrm{E}-1178 \mathrm{~m}$ alt.) développé sur le piémont de l'éperon de Tadmor témoigne de la mobilité des versants de la montagne en lien avec les premières populations d'agropasteurs. Constitué par la superposition de quatre phases de pédogenèse, le profil a enregistré jusqu'à $4 \mathrm{~m}$ d'histoire pédosédimentaire sur le versant. Les sols sont développés sur des dépôts meubles, épais, constitués de limons sablo-argileux sans changement dans le régime hydrosédimentaire révélé par la texture fine. Les horizons organo-minéraux supérieurs de deux premiers paléosols ont été identifiés respectivement vers 3,30 m (Ab3) et vers 2,50 m (Ab2). De texture argilo-sableuse, ces horizons supérieurs sont bien structurés en agrégats prismatiques à columnaires sur une épaisseur très conséquente dans les trois paléosols ( $40 \mathrm{~cm}$ pour $\mathrm{Ab} 1)$. Ils sont riches en matière organique qui leur confère une couleur sombre ainsi qu'en charbons de bois dispersés dans toute leur épaisseur. Ces sols témoignent de l'importance des changements liés à la couverture forestière, à la puissance de l'érosion et de la capacité de transport du ruissellement, particulièrement dans le paléosol le plus ancien. Ce dernier se distingue également par la limite inférieure de son horizon organo-minéral qui présente de profondes pénétrations en langues (jusqu'à $20 \mathrm{~cm}$ ) dans la roche-mère meuble. Une datation effectuée sur un des plus gros charbons a fourni un terminus post quem pour les apports postérieurs, vers $4934-4726$ cal. BC (5950 40 BP ; Poz-54645). Le recouvrement de ce sol 
très évolué par des apports rapides suggère une extrême fragilisation des sols sur le piémont du relief de Tadmor durant le Chalcolithique Moyen (Néolithique récent de Byblos), voire sur le plateau sommital lui-même. La troisième phase de stabilité, marquée par une reprise de pédogenèse, correspond à l'horizon de surface du paléosol le moins enfoui (Ab1) et relativement épais $(0,60 \mathrm{~m})$. Il forme avec l'horizon M1 un paléosol Ab1+M1 de 1,70 m d'épaisseur. L'horizon M1 est en relation avec un empierrement anthropique de $80 \mathrm{~cm}$ d'épaisseur, constitué de cailloux de taille hétérogène mêlés à des graviers. Les charbons de bois prélevés dans les horizons de ce paléosol datent de la première moitié du III ${ }^{e}$ millénaire $\mathrm{BC}$ avec des âges (AMS ${ }^{14} \mathrm{C}$ ) entre $4050 \pm 35$ BP soit vers $2839-2473$ cal. BC (Poz-67391) et 4180 \pm 40 BP soit vers 2891-2631 cal. BC (Poz-76314). Des fragments de céramique glaçurée, striée au peigne avant cuisson, très usés, ont été retrouvés dans l'aménagement anthropique, ce qui confirme une certaine durée d'évolution de ce sol avant son enfouissement (au début du Moyen Âge ?). Cette hypothèse est démontrée par l'observation pédologique macroscopique de sa structure.

Tous les sondages pédoarchéologiques réalisés dans la haute vallée du Nahr Ibrahim vont dans le sens d'une longue histoire des paysages. Ils témoignent, à des degrés divers, des effets combinés des pratiques agropastorales et des variables naturelles dans la mobilité de la couverture pédosédimentaire et dans la qualité des sols. L'importance de la dégradation du couvert végétal à la fin du Néolithique se mesure à leurs conséquences dans certaines unités paysagères sans pour autant préciser l'état du couvert arboré local à ce moment, son étendue, la qualité des essences et leur distribution. Les données paléoécologiques relatives aux espaces intérieurs du Liban s'accordent sur la composition générale de la végétation ligneuse postglaciaire, à partir des analyses palynologiques réalisées dans le polje de Yammouneh (1 $360 \mathrm{~m}$ alt.) à l'est du bassin versant du Nahr Ibrahim, ainsi que les carottages de Ammiq et de Chamsine/ Anjar dans la Béqaa méridionale (850 $\mathrm{m}$ alt.) et en haute montagne $(2100 \mathrm{~m})$, au nord de notre région d'étude, au lieu-dit Al Jourd (Gasse, et al., 2011 ; Hajar, et al., 2010 ; Chedadi et Khater, 2016). Ces enregistrements rendent compte de cortèges végétaux dominés par la chênaie suivie du cèdre (Cedrus libani), du pin (Pinus halepensis), du sapin (Abies sp.), des Cupressacées (dont Juniperus sp.), du pistachier (Pistacia sp.) et de l'olivier (Olea europaea). La distribution spatiale et l'évolution temporelle des taxons arborés au début de l'Holocène varient sensiblement, non seulement selon les conditions altitudinales mais également au plan micro-régional dans la Béqaa méridionale (наjar et al., 2010). L'expansion forestière sur les versants du Mont Liban, concomitante d'une augmentation des températures et des précipitations hivernales, amorcée à la fin du Dryas récent, est effective dès 11000 cal. BP vers $850 \mathrm{~m}$ et antérieurement à 5000 cal. BP aux altitudes inférieures à $2100 \mathrm{~m}$ (Chedadi et Khater, 2016).

Dans le bassin versant du Nahr Ibrahim, la montagne est composée d'une mosaïque de milieux, très peu étendus spatialement pour certains, dont les caractéristiques lithologiques, édaphiques et hydrologiques permettent d'offrir des conditions d'installation durable à des groupes d'agropasteurs. Le milieu, malgré les contraintes imposées par l'altitude pour l'implantation de l'habitat et pour la recherche de subsistance, est suffisamment riche en sols et en eau. Les semailles d'automne, les cycles végétatifs et les phyllothermes de la plupart des céréales sont compatibles avec les données climatiques jusqu'à $2000 \mathrm{~m}$ d'altitude. Le réchauffement du climat associé 
à une augmentation des précipitations qui est observé au début de l'Holocène au Proche-Orient est enregistré au Liban dans les spéléothèmes de Jiîta, par l'analyse multi-proxy des sédiments de l'alvéole d'effondrement de Yammouneh et par les pollens fossiles de la Béqaa, tandis qu'un microclimat plus froid est restitué au-dessus de $2000 \mathrm{~m}$ où les températures seraient de 2 à $4^{\circ} \mathrm{C}$ inférieures à l'actuel jusque vers 5000 cal.BP (Roberts, et al., 2017 ; Verheyden, et al., 2008 ; Cheng, et al., 2015 ; Gasse, et al., 2011 ; 2015 ; Hajar, et al., 2010 ; Chedadi et Khater, 2016). Une amélioration des conditions climatiques a pu aussi contribuer à la fixation du peuplement en altitude. Un climat globalement plus chaud et plus humide vers le milieu de l'Holocène, autour de 7000 BP et jusqu'à $6000 \mathrm{BP}$, avant le début d'une phase régionale plus sèche vers $5300 \mathrm{BP}$ (Nader, et al., 2007 ; Verheyden, et al., 2008 ; Cheng, et al., 2015 ; Develle, et al., 2010 ; Roberts, et al., 2011), favorise une certaine stabilité de la production agricole. Les interventions sur la couverture végétale dans la haute vallée du Nahr Ibrahim, vers $1400 \mathrm{~m}$ d'altitude, au début du Ve millénaire, s'inscrivent dans une tendance amorcée un millénaire plus tôt sur le versant oriental du Mont Liban où des déforestations localisées sont perceptibles dès le $\mathrm{VI}^{\mathrm{e}}$ millénaire par des populations d'agropasteurs occupant déjà la plaine de la Béqaa (Hajar, et al., 2010). Les études récentes qui tentent d'évaluer l'impact des changements climatiques entre 9500 et 7500 cal. BP sur le comportement socioéconomique des premières communautés d'agropasteurs au Proche-Orient soulignent la diversité des réponses sociétales au plan micro-régional ainsi que les capacités d'adaptation et de résilience au stress environnemental des populations. Cette résilience s'exprime sur une durée variable en fonction des conditions systémiques (Flohr, et al., 2016 ; Roberts, et al., 2017). Il faut d'ailleurs ajouter aux conditions systémiques, la prise en considération des situations conjoncturelles surtout aux époques historiques. Il n'en demeure pas moins que l'approche adoptée dans cette étude comme dans les nouvelles études pluridisciplinaires nuancent sensiblement les précédentes approches binaires simplistes (RCC-Rapid Climatic Changes = basculement culturel ex abrupto; collapse) fondées sur: i) l'utilisation de données archéologiques et de sources écrites hétéroclites souffrant d'importantes disparités régionales ainsi que d'une qualité/fiabilité très inégale, ii) des sites de référence à partir de fouilles très anciennes à l'enregistrement stratigraphique discutable (Weininger, et al., 2009 ;Kaniewski, et al., 2013) ou iii) la manipulation des données archéologiques (Braemer, 2014 ; Genz, 2015).

Dans la haute vallée du Nahr Ibrahim, la fouille archéologique a permis de démontrer l'existence d'un habitat de montagne sur le Tell el-Kharayeb dès l'aube du III ${ }^{e}$ millénaire, les dates ${ }^{14} \mathrm{C}$ obtenues étant comprises entre $4430 \pm 35 \mathrm{BP}$ (soit vers $3328-2925 \mathrm{cal}$. BC ; Poz-76217) et $4300 \pm 35$ BP (soit vers $3012-2880$ cal. BC; Poz-76216) (Harfouche, et al,, 2017). Cet habitat est comparable aux sites de la côte syro-libanaise de l'âge du Bronze Ancien par son architecture ainsi que par ses modes de production alimentaires et artisanaux. Si la singularité de la montagne dans ses formes de peuplement et d'aménagement du territoire reste à caractériser précisément, les résultats montrent d'ores et déjà que les reliefs au-dessus de $1000 \mathrm{~m}$ sont peuplés par des communautés sédentaires et organisées, dès le début de l'âge du Bronze Ancien (agriculture en terrasses, potiers locaux et production céramique intensive standardisée). La montagne n'est pas uniquement un lieu de passage entre la plaine littorale et la Béqaa. L'étude du mobilier céramique en particulier démontre que ce territoire en altitude est parfaitement intégré aux réseaux des échanges avec les régions voisines et à plus longue distance (Levant Sud, Égypte), dès l'aube du III millénaire (Baldi, 2017). 
Les fouilles archéologiques réalisées derrière les murs de terrasses actuels dans l'environnement du Tell el-Kharayeb ont révélé la présence de champs antérieurs, également soutenus par des murs construits en pierre sèche. L'étude pédologique macroscopique réalisée sur le terrain est complétée par des prélèvements de sédiments et de charbons de bois dans les horizons des sols/paléosols en relation avec ces terrasses, pour des analyses physico-chimiques $\left(\mathrm{CaCO}_{3}\right.$ actif ; matière organique ; $\mathrm{pH}$; Azote-N ; phosphore-P ; potassium-K ; sédimentologie), de phytholithes et des datations ${ }^{14} \mathrm{C}$ en AMS. Cette archéologie du champ, nouvelle pour la recherche au Liban, a démontré une phase de mise en culture précoce, contemporaine de l'habitat, au début du III millénaire (Harfouche et Poupet, 2002; Harfouche, 2007; Harfouche, et al., 2015a). L'aménagement de la montagne en terrasses par les sociétés villageoises dès l'âge du Bronze Ancien à la suite de défrichements a produit un fort impact sur l'environnement forestier, les sols et les ressources en eau (Harfouche, et al., 2015b). Dans les reliefs accidentés, comme en montagne, la pérennité de l'habitat passe par une véritable construction de l'espace de la production agricole. Les échantillons prélevés dans les sols et les paléosols des sondages, qui ont fait l'objet d'analyses de phytolithes, ont permis de caractériser les espèces végétales (graminées, dicotylédones, pinacées) disponibles dans l'environnement à l'Holocène et les plantes cultivées associées aux paléosols tels l'orge et le blé (Verdin, 2015). L'analyse carpologique des macro-restes végétaux présents dans l'habitat du Tell el-Kharayeb identifie la présence de l'orge vêtue à deux rangs (Hordeum distichum), majoritaire, du blé engrain (Triticum monococcum) et l'amidonnier ( $T$. dicoccum), des légumineuses cultivées, lentille (Lens culinaris) et ers (Vicia ervilia), ainsi que la vigne (Vitis vinifera) et l'olivier (Olea europaea) sur les versants de la haute vallée, dès le début de l'âge du Bronze Ancien (Hevreux, 2017). L'olivier est encore présent aujourd'hui parmi les cultures en terrasses des communes de Mghaïra et de Mejdel par exemple, dans un milieu montagneux proche de sa limite altitudinale supérieure de culture.

36 Le caractère agricole du paysage aménagé en terrasses autour de l'habitat est précisé par la présence des carporestes de plantes cultivées et des adventices de culture (ex. Galium sp. et Lolium sp.), ainsi que par l'outillage en silex dont des lames-faucilles (Yazbeck, 2017). Sur le plan des activités pastorales, les analyses de paléosols montrent un amendement phosphaté qui renvoie à la pratique de la fumure sur les champs en terrasses localisés au sud du tell et permet de constater une présence du troupeau à l'âge du Bronze Ancien (Harfouche, et al., 2015a), ce dernier étant majoritairement constitué de Caprinae d'après l'ostéofaune (Chahoud inédit). Si elles n'avaient pas développé une approche érudite des mécanismes de l'érosion, les populations anciennes avaient conscience de ces phénomènes puisqu'elles ressentaient déjà le besoin de protéger et d'améliorer les sols.

Un paysage se dessine progressivement, constitué de champs en terrasses identifiés dans l'environnement de l'habitat, de milieux ouverts non cultivés comprenant des espèces fruitières arbustives et sauvages comme l'amandier (Amygdalus sp.), et de peuplements d'arbres résineux exploités comme ressource en bois pour l'architecture. Les premières données anthracologiques soulignent l'importance des conifères dont le cyprès (Cupressus sempervirens L.), ce qui est conforme avec les analyses de phytolithes qui révèlent aussi la présence du pin dans l'environnement du site (Hevreux, 2017 ; Verdin, 2015). Une des particularités des espaces de l'habitat mis au jour est l'utilisation de la terre crue comme matériau principal des bâtisseurs au Bronze Ancien 
et qui est étroitement associée au bois dans l'architecture. La présence de pollens de Cupressacées (particulièrement le genévrier/Juniperus) a été observée en amont du bassin versant $\mathrm{du}$ Nahr Ibrahim, dans les dépôts sédimentaires quaternaires de Yammouneh (Gasse, et al., 2011). Toutefois, l'évolution de la distribution de ces essences au cours de l'Holocène demeure inconnue car les pollens postérieurs à $8300 \mathrm{BP}$ sont absents des enregistrements en raison des conditions taphonomiques. S'il n'est pas possible de spatialiser les groupements forestiers dans la haute vallée du Nahr Ibrahim et ses abords à ce stade, l'utilisation quasi-exclusive du cyprès dans la construction des structures (cloison, toiture) d'un espace habité qui ne présente aucun signe d'une singularité sociale ou économique, suggère que les habitants-constructeurs avaient accès à courte distance à ce taxon ligneux. Les restes fauniques témoignent de la présence d'espèces forestières (cervidé) dans l'environnement du Tell el-Kharayeb. Ces premiers résultats sont essentiels pour la connaissance du couvert végétal de la montagne de l'arrière-pays de Byblos à une époque directement antérieure au corpus de textes et d'images abondamment étudié de la fin du $\mathrm{III}^{\mathrm{e}}$ et du $\mathrm{II}^{\mathrm{e}}$ millénaire BC.

Les millénaires suivants ( $\mathrm{du} \mathrm{I}^{\mathrm{er}}$ millénaire $\mathrm{BC}$, jusqu'à la période médiévale) dans la haute vallée du Nahr Ibrahim seront marqués par la mobilité des paysages en terrasses, au travers des périodes de leur fort développement et puis de leur déclin partiel (Harfouche, et al., 2015a ; 2017). Durant les périodes caractérisées par un climat plus sec, de 5300 à 4200 BP et puis vers 2800 et 1400 BP (Cheng, et al., 2015 ; Roberts, et al., 2017) ayant pu influer directement sur la qualité des récoltes, la spécificité hydroclimatique du Mont Liban a pu jouer un rôle grâce à l'humidité maintenue sur les reliefs du Mont Liban. Par ailleurs, la brutale et (relativement) courte phase climatique plus sèche qui aurait été responsable du supposé collapse de la fin de l'âge du Bronze vers 3200 BP soit vers $1200 \mathrm{BC}$ (Kaniewski, et al., 2013) au Proche-Orient correspond, au contraire, à une longue période humide entre 4100 et $2900 \mathrm{BP}$ sur les reliefs du Mont-Liban (Cheng, et al., 2015 ; Develle, et al., 2010).

\section{4 - Conclusion}

Durant les cinq derniers millénaires, des continuités et des ruptures apparaitront dans la dialectique sociétés-milieux, mais les phases d'abandon et de reconstruction sous l'effet des dynamiques pédosédimentaires et de l'évolution du peuplement s'inscrivent dans une trajectoire fortement marquée par cette première structuration des territoires habités de la montagne. La résilience face au stress environnemental est appuyée par nos travaux qui montrent un fort ancrage territorial des sociétés en montagne, dès $2900 \mathrm{BC}$, sans interruption majeure, tant sur le plan de l'habitat villageois que du parcellaire agricole en pierre sèche. Dès lors que les communautés d'agriculteurs se constituent, avec une empreinte de plus en plus affirmée sur le territoire, une complexification sociale grandissante ainsi qu'un héritage technique et socio-culturel, la réactivité aux stimuli externes d'origine naturelle est modifiée et l'enregistrement à l'échelle locale devient déterminant, surtout dans un espace contraignant. Les réponses aux changements se sont exprimées selon des temporalités multiples (Harfouche, et al., 2016). Depuis le début du III millénaire BC, les sociétés montrent leur capacité à faire évoluer le modèle socioéconomique de l'organisation villageoise, en particulier grâce au dynamisme de l'hinterland montagneux induit par le lien jamais rompu avec les forces vives exogènes : circuits économiques actifs avec le 
littoral, production locale de céramiques et d'outils intégrée aux réseaux des échanges (emprunts techniques et importations), exploitation marchande des lieux de culte/ pèlerinage depuis le $\mathrm{I}^{\mathrm{er}}$ millénaire BC (Harfouche, et al., 2017; Yazbeck, 2017; Baldi, 2017 ; Homsy-Gottwalles, 2017 ; Zaven, 2017).

\section{BIBLIOGRAPHIE}

Abdallah, Ch., N. Baghdadi, et R. Ziadé. 2015. Évaluation de l'instabilité historique des terrains au Liban : le cas du Nahr Ibrahim. In Du Mont Liban aux Sierras d'Espagne : sols, eau et sociétés en montagne. Autour du projet franco-libanais CEDRE "Nahr Ibrahim", ed. Archaeopress, 51-72. Oxford.

Baldi, J.S. 2017. La céramique de l'âge du Bronze Ancien du Tell el-Kharayeb. In : Paysages et peuplement du Mont Liban. La vallée du Nahr Ibrahim et l'hinterland de Byblos, ed. Ministère de la Culture du Liban, 219-246. Beyrouth.

Braemer, F. 2014. Compte-rendu de Harvey WEISS (éd.), Seven Generations Since the Fall of Akkad. Syria 91: 462-466.

Brun, J.-P. 2004. Archéologie du vin et de l'huile. De la préhistoire à l'époque hellénistique, Paris: Errance.

Cheddadi, R., and C. Khater. 2016. Climate change since the last glacial period in Lebanon and the persistence of Mediterranean species. Quaternary Science Reviews 150: 146-157.

Cheng, H., A. Sinha, S. Verheyden, F.H. Nader, X.L. Li, P.Z. Zhang, J.J. Yin, L. Yi, Y.B. Peng, Z.G. Rao, Y.F. Ning, and R.L. Edwards. 2015. The climate variability in northern Levant over the past 20,000 years. Geophysical Research Letters 42: 8641-8650. doi:10.1002/2015GL065397.

Copeland, L., and P.J. Wescombe. 1965. Inventory of Stone-Age Sites in Lebanon. Mélanges de l'Université Saint-Joseph 41(2): 29-175.

Daëron, M., Y. Klinger, P. Taponnier, A. Elias, E. Jacques, and A. Sursock. 2007. 12,000-Year-Long Record of 10 to 13 Paleoearthquakes on the Yammoûneh Fault, Levant Fault System. Lebanon. Bulletin of the Seismological Society of America 97(3): 749-771.

Develle, A.-L., J. Herreros, L. Vidal, A. Sursock, and F. Gasse. 2010. Controlling factors on a paleolake oxygen isotope record (Yammoûneh, Lebanon) since the Last Glacial Maximum. Quaternary Science Reviews (29): 865-886.

Doumet-Serhal, C. 2010. Jabal Moussa : archaeological survey. In Jabal Moussa entre mythe et réalité à travers 22 témoignages, éd. Association pour la Protection du Jabal Moussa, 19-23, Liban.

Dubertret, L. 1951. Carte géologique au 50.000 . Feuille de Qartaba. Beyrouth: Ministère des travaux publics de la République Libanaise.

Elayi, J. 1988. L'exploitation des Cèdres du Mont Liban par les rois assyriens et néo-babyloniens. Journal of the Economic and Social History of the Orient 31(1): 14-41.

Flohr, P., D. Fleitmann, R. Matthews, W. Matthews, and S. Black. 2016. Evidence of resilience to past climate change in Southwest Asia: Early farming communities and the 9.2 and 8.2 ka events. Quaternary Science Reviews 136: 23-39. 
Fontan, E. 2001. La frise du transport du bois, décor du palais de Sargon II à Khorsabad. Archaeology and History in the Lebanon 14: 58-63.

Gasse, F., L. Vidal, A.-L. Develle, and E. Van Campo. 2011. Hydrological variability in the Northern Levant: a 250 ka multiproxy record from the Yammoûneh (Lebanon) sedimentary sequence. Clim. Past 7: 1261-1284.

Gasse, F., L. Vidal, E. Van Campo, F. Demory, A.-L. Develle, K. Tachikawa, A. Elias, E. Bard, M. Garcia, C. Sonzogni, and N. Thouveny. 2015. Hydroclimatic changes in northern Levant over the past 400,000 years. Quaternary Science Reviews 111: 1-8.

Gatier, P.-L., et L. Nordiguian dir. 2005. Yanouh et le Nahr Ibrahim. Nouvelles découvertes archéologiques dans la vallée d'Adonis. Beyrouth: Presses de l'Université Saint-Joseph.

Genz, H. 2015. Beware of environmental determinism: the transition from the Early to the Middle Bronze Age on the Lebanese coast and the $4.2 \mathrm{ka}$ BP event. In 2200 BC - A climatic breakdown as a cause for the collapse of the old world? ed. Landesamt für Denkmalpflege und Archäologie SachsenAnhalt, Landesmuseum für Vorgeschichte, 97-111. Halle (Saale).

Guidoboni, E., A. Comastri, and G. Traina. 1994. Catalogue of Ancient Earthquakes in the Mediterranean Area up to the $10^{\text {th }}$ Century. Bologna: ING, Roma-SGA.

Hajar, L., M. Haïdar-Boustani, C. Khater, and R. Cheddadi. 2010. Environmental changes in Lebanon during the Holocene: Man vs. climate impacts. Journal of Arid Environments 74(7): 746-755.

Harfouche, R. 2003. Le Père Joseph Delore, un observateur attentif de la nature et de la vie rurale. In Les "petites écoles" du Mont-Liban. Joseph Delore s. j. (1873-1944), éd. Presses de l'Université SaintJoseph, 66-85. Beyrouth.

Harfouche, R. 2007. Histoire des paysages méditerranéens terrassés : aménagements et agriculture. Oxford: Archaeopress.

Harfouche, R., et P. Poupet. 2001. Yanouh. Les formes du paysage : histoire et exploitation des ressources minérales et des sols. Bulletin d'Archéologie et d'Architecture Libanaises 5: 132-137.

Harfouche, R., et P. Poupet. 2002. Les sondages dans les terrasses agricoles. Bulletin d'Archéologie et d'Architecture Libanaises 6: 230-234.

Harfouche, R., et P. Poupet. 2009. Five Thousand Years of Soil Care and Land Use at the Springhead of Nahr Ibrahim (Mount Lebanon): A Multidisciplinary View from Archaeology and Pedology. In 10th International Meeting on Soils with Mediterranean Type of Climate: Soil Conservation and Protection is the Basis for Sustainable Land, ed. CNRSL/IUSS, 155-160. Beirut.

Harfouche, R., et P. Poupet (textes réunis par). 2015. Du Mont Liban aux Sierras d'Espagne : sols, eau et sociétés en montagne. Autour du projet franco-libanais CEDRE "Nahr Ibrahim". Oxford: Archaeopress.

Harfouche, R., P. Poupet, T. Darwich, P. Verdin, A. Shaban, C. Khater, T. Goslar, et G. Faour. 2015a. Nouveaux regards multidisciplinaires sur la montagne de l'hinterland de l'antique Byblos (Jbail). In : Du Mont Liban aux Sierras d'Espagne : sols, eau et sociétés en montagne. Autour du projet francolibanais CEDRE "Nahr Ibrahim", ed. Archaeopress, 73-11. Oxford.

Harfouche, R., P. Poupet, C. Khater, et T. Darwich. 2015b. Forêts, sols et sociétés : une longue histoire commune. In : Du Mont Liban aux Sierras d'Espagne : sols, eau et sociétés en montagne. Autour du projet franco-libanais CEDRE "Nahr Ibrahim", ed. Archaeopress, 125-136. Oxford.

Harfouche, R., P. Poupet, T. Darwich, A. Shaban, P. Verdin, G. Faour, C. Khater, et A. Assaker. 2016. La construction des territoires agraires et la forêt dans la montagne libanaise : impacts 
environnementaux et enjeux sociaux depuis l'Antiquité. In Aménagement et environnement, éd. Presses Universitaires de Rennes, 75-89. Rennes.

Harfouche, R., avec la collaboration de P. Poupet, J.S. Baldi, C. Yazbeck, C. Abdallah, L. Herveux, G. Homsy-Gottwalles, M. Abboud, et T. Zaven. 2017. Paysages et peuplement du Mont Liban. La vallée du Nahr Ibrahim et l'hinterland de Byblos. Beyrouth: Ministère de la Culture du Liban.

Herveux, L. 2017. Les végétaux récoltés et exploités. In : Paysages et peuplement du Mont Liban. La vallée du Nahr Ibrahim et l'hinterland de Byblos, ed. Ministère de la Culture du Liban, 246-250. Beyrouth.

Homsy-Gottwalles, G. 2017. La céramique et le mobilier métallique. In : Paysages et peuplement du Mont Liban. La vallée du Nahr Ibrahim et l'hinterland de Byblos, ed. Ministère de la Culture du Liban, 256-265. Beyrouth.

Huot, J.-L. 2004. Une archéologie des peuples du Proche-Orient. I : Des premiers villageois aux peuples des cités-États (X $\mathrm{X}^{e}-I I I^{e}$ millénaire av. J.-C.). II : Des hommes des Palais aux sujets de premiers empires (II ${ }^{e} I^{e r}$ millénaire av.J.-C.). Paris: Errance.

Jacomet, S. et alii. 2016. On-site data cast doubts on the hypothesis of shifting cultivation in the late Neolithic (c. 4300-2400 cal. BC) : Landscape management as an alternative paradigm. The Holocene 26(11): 1858-1874.

Kaniewski, D., E. Van Campo, J. Guiot, S. Le Burel, Th. Otto, and C. Baeteman. 2013. Environmental Roots of the Late Bronze Age Crisis. PLoS ONE 8(8): e71004.

Nader, F.H., S. Verheyden, H. Cheng, et R. Swennen. 2007. Étude pétrographique et géochimique d'une stalagmite de la grotte de Jeita (Liban). Lebanese Science Journal 8(2): 45-58.

Nehmé, C., S. Verheyden, S.R. Noble, A.R. Farrant, D. Sahy, J. Hellstrom, J.-J. Delannoy, and P. Claeys. 2015. Reconstruction of MIS 5 climate in the central Levant using a stalagmite from Kanaan Cave, Lebanon. Climate of the Past 11: 1785-1799.

Roberts, N., W.J. Eastwood, C. Kuzucuoglu, G. Fiorentino, and V. Caracuta. 2011. Climatic vegetation and cultural change in the eastern Mediterranean during the mid-Holocene environmental transition. The Holocene 21: 147-162.

Roberts, N., J. Woodbridge, A. Bevan, A. Palmisano, S. Shennan, and E. Asouti. 2017. Human responses and non-responses to climatic variations during the last Glacial-Interglacial transition in the eastern Mediterranean. Quaternary Science Reviews vol.in press: 1-21.

Sbeinati, M. R., R. Darawcheh, and M. Mouty. 2005. The historical earthquakes of Syria: an analysis of large and moderate earthquakes from 1365 B.C. to 1900 A.D. Annals of Geophysics 48(3): 347-435.

Shaban, A., T. Darwich, A. Assaker, P. Poupet, et R. Harfouche. 2015. Évaluation des caractéristiques physiques et des risques naturels dans le bassin versant du Nahr Ibrahim. In : Du Mont Liban aux Sierras d'Espagne : sols, eau et sociétés en montagne. Autour du projet franco-libanais CEDRE "Nahr Ibrahim", ed. Archaeopress, 739-50. Oxford.

Shaban, A., and M. Hamze. 2017. Shared water resources of Lebanon. New York: Nova science publishers.

Verdin, P. 2015. Essai de caractérisation de sols cultivés de l'âge du Bronze sur le site de tell elKharayeb, Liban. Évaluation du potentiel de l'analyse de phytolithes. In : Du Mont Liban aux Sierras d'Espagne : sols, eau et sociétés en montagne. Autour du projet franco-libanais CEDRE "Nahr Ibrahim", ed. Archaeopress, 111-113. Oxford. 
Verheyden, S., F.H. Nader, H.J. Cheng, L.R. Edwards, and R. Swennen. 2008. Paleoclimate reconstruction in the Levant region from the geochemistry of a Holocene stalagmite from the Jeita cave, Lebanon. Quaternary Research 70: 368-381.

Weninger, B. et alii. 2009. The Impact of Rapid Climate Change on prehistoric societies during the Holocene in the Eastern Mediterranean. Documenta Praehistorica 36: 7-59.

Yazbeck, C. 2017. L'outillage en silex. In : Paysages et peuplement du Mont Liban. La vallée du Nahr Ibrahim et l'hinterland de Byblos, ed. Ministère de la Culture du Liban, 250-252. Beyrouth.

Zaven, T. 2017. Les verres. In : Paysages et peuplement du Mont Liban. La vallée du Nahr Ibrahim et l'hinterland de Byblos, ed. Ministère de la Culture du Liban, 269-270. Beyrouth.

\section{RÉSUMÉS}

Le projet de recherche franco-libanais "Nahr Ibrahim" initié autour de la vallée éponyme de ce fleuve majeur du Mont-Liban s'attache à caractériser les impacts sur l'environnement forestier, les sols et les ressources en eau de la construction des paysages en terrasses, et des formes de peuplement à l'Holocène. La définition des transitions et les temporalités des changements sont examinées à partir des données archéologiques et historiques, radiochronologiques $\left({ }^{14} \mathrm{C}\right)$, géologiques et pédologiques, paléobotaniques (anthracologie, carpologie, phytolithes), ethnologiques et iconographiques (dont les photographies du début du $\mathrm{XX}^{\mathrm{e}} \mathrm{s}$. par les Pères Jésuites). L'étude du bassin versant du Nahr Ibrahim est articulée à une approche multiscalaire $\mathrm{du}$ territoire entre le Nahr el-Kelb au sud et le Nahr ej-Jaouz au nord, pour cartographier les variations de l'occupation du sol. Dans cet article, nous discutons les apports et les limites de la carte archéologique puis nous mettons en perspective l'étude d'un profil de sol polygénique plurimétrique avec les premiers résultats provenant de la fouille de l'habitat sédentaire le plus ancien documenté à ce jour dans la montagne libanaise. L'approche intégrative met en évidence des réponses de l'environnement à l'exploitation des ressources par les sociétés et des adaptations de ces dernières aux changements dans la seconde moitié de l'Holocène, depuis les défrichements vers $6000 \mathrm{BP}$ jusqu'aux mutations paysagères et socio-économiques récentes.

The French-Lebanese Nahr Ibrahim Project was undertaken on the valley of this major river in Mount Lebanon. It aims to characterize the environmental impacts on forests, soils and water resources of landscape terracing (a typical feature of Mount Lebanon), and of settlement patterns in the Holocene. The definition of transitional periods and the timescales of changes are examined through archaeological and historical data, ${ }^{14} \mathrm{C}$ datings, geology and soil science (paleosols), paleobotany (anthracology, carpology, phytholits), anthropology and ancient photographs (due to the Jesuit Fathers in the beginnings of the XX c.). The study of the Nahr Ibrahim watershed is linked to a multiscalar approach of the territory lying between Nahr elKelb toward south and Nahr ej-Jaouz toward north in order to map the variations in land-use. The present paper focuses on the strengths and weaknesses of the archaeological data base to achieve this mapping. We then expose the main aspects of the study of a four meters polygenetic soil profile in regard to the first records from a recent excavation on the most ancient sedentary settlement known in the mountain. The integrative approach brings into light the environmental answers to the exploitation of natural resources and in return the adaptation of societies to these variations in the second half of the Holocene, from the forest clearing around $6000 \mathrm{BP}$ up to recent landscape and socio-economical changes. 
INDEX

Index géographique : Liban, Nahr Ibrahim, Nahr el-Kelb, Nahr ej-Jaouz, Byblos, Yanouh, Mghaïra

Keywords : Mount Lebanon, agricultural terraces, geoarchaeology, Holocene

Mots-clés : Mont-Liban, terrasses agricoles, géoarchéologie, Holocène

\section{AUTEURS}

ROMANA HARFOUCHE

UMR 7041 CNRS, ArScAn, Nanterre, France

PIERRE POUPET

Centre national de la recherche scientifique, France

CHADI ABDALLAH

Centre de Télédétection, Cnrs-Liban

LINDA HERVEUX

UMR 5133 Archéorient, Lyon, France

CORINE YAZBECK

Département d'arts et d'archéologie, faculté des beaux-arts, Université Libanaise, Liban GHALEB FAOUR

Centre de Télédétection, CNRS-Liban

JOHNNY SAMUELE BALDI

Institut français du Proche-Orient, Beyrouth, Liban

JWANA CHAHOUD

Département d'arts et d'archéologie, faculté des beaux-arts, Université Libanaise, Liban MOHAMMAD ABBOUD

School of Engineering, Lebanese International University, Lebanon

GRACE HOMSY-GOTTWALLES

Département d'arts et d'archéologie, faculté des beaux-arts, Université Libanaise, Liban

\section{TANIA ZAVEN}

Direction générale des antiquités du Liban

PASCAL VERDIN

INRAP et UMR 7264 CNRS, CÉPAM, Nice, France 